\title{
The role of immature granulocytes and inflammatory hemogram indices in the inflammation
}

\author{
Said Incir ${ }^{1}$, (1) Hatice Kant Calti' ${ }^{1}$, (1) Kerim Erhan Palaoglu ${ }^{2}$
}

'Department of Laboratory, Koc University Hospital, Istanbul, Turkey

${ }^{2}$ Department of Laboratory, American Hospital, Istanbul, Turkey

\begin{abstract}
Objectives: In this retrospective study, we aimed to assess the role of immature granulocyte percentage (IG\%), inflammatory complete blood count (CBC) parameters and indices in investigating the severity of inflammation, to evaluate their correlation, and to determine their predictive ability in classifying inflammation.

Methods: We obtained hematological and biochemical data of the 161 outpatients for this study. Patients were assigned to three groups according to their C-reactive protein (CRP) levels. Group I had a CRp-value of $<3 \mathrm{mg} / \mathrm{L}$ (non-inflammatory group) ( $n=58$ ), Group II had a CRP level between 3 to 9mg/L (low-grade inflammatory group) ( $n=59)$, and Group III had a CRP level of $>9 \mathrm{mg} / \mathrm{L}$ (clinically significant inflammatory group)( $\mathrm{n}=44)$. The between-group differences were evaluated concerning sex, age, CRP, procalcitonin, IG\%, CBC parameters and indices, including Platelet-to-Lymphocyte Ratio (PLR), Neutrophil-to-Lymphocyte Ratio (NLR) and Systemic Immune-Inflammatory Index (SII).

Results: In Group II, the levels of CRP ( $p<0.0001)$, platelet count $(p<0.05)$, PLR $(p<0.05)$, NLR $(p<0.05)$ and SII $(p<0.01)$ were significantly higher than those in the Group I. In Group III, CRP $(p<0.0001)$, IG\% ( $<<0.0001)$, procalcitonin ( $p<0.01)$, platelet count $(p<0.05)$, PLR $(p<0.01)$, NLR $(p<0.05)$ and SII $(p<0.01)$ values showed a significant between-group difference when compared to Group I. A significant difference between Group II and Group III was detected for, CRP $(p<0.0001)$ and IG\% $(p<0.05)$. There were significant positive correlations among IG\% and CRP $(p<0.001)$, platelet count $(p<005)$, PLR $(p<0.05)$ and SII $(<0.05)$. The sensitivity and specificity values of the IG\% using a cut-off value of $>0.2$ were $75.3 \%$ and $52.5 \%$, respectively.

Conclusion: The findings obtained in this study suggest that to detect the severity of inflammation, it would be more reliable to evaluate the combination of $C B C$ parameters with biochemical markers instead of looking at a single one.

Keywords: Automated blood cell counters, hematology, immature granulocytes, inflammation
\end{abstract}

nflammation should be rapidly detected and differentiated to determine the underlying pathology. It is vital to distinguish any infection from other causes of inflammation because of early intervention. If a physician suspects of inflammation or infection after a physical examination, biochemical markers or blood cultures are needed for making a correct diagnosis. As these are time-consuming and not cost-effective, faster and cheaper indicators are required for early intervention $[1,2]$. In this regard, inflammatory markers, such as C-re- active protein (CRP) and procalcitonin, have been routinely used for the early diagnosis [3].

By the development of new hematology analyzers, the early detection of inflammation has become possible. Recent studies have pointed out that leukocyte count, absolute neutrophil count (ANC) and band count may be an early indicator of inflammation or sepsis [4]. The Sysmex XN-3100 (Sysmex Corporation, Kobe, Japan) enables to detect the percentage of immature granulocytes (IG\%) using flow cytometry in the

Address for correspondence: Said Incir, MD. Department of Laboratory, Koc University Hospital, Istanbul, Turkey

Phone: +90 5322183766 E-mail: sincir@kuh.ku.edu.tr ORCID: 0000-0002-7700-7388

Submitted Date: March 24, 2020 Accepted Date: June 06, 2020 Available Online Date: October 05, 2020

${ }^{\circ}$ Copyright 2020 by International Journal of Medical Biochemistry - Available online at www.internationalbiochemistry.com

OPEN ACCESS This work is licensed under a Creative Commons Attribution-NonCommercial 4.0 International License. 
DIFF-channel. Forward-scattered light, lateral-scattered light, and lateral fluorescent light are used to detect volume, complexity, and DNA/RNA content [5].

Immature granulocyte count refers to the sum of promyelocyte, myelocyte and metamyelocyte cells that mature along with the myeloid series from the multipotent stem cell located in the bone marrow. Myelocytes and metamyelocytes are precursor forms of granular leukocytes, defined as band neutrophils and segmented neutrophils in peripheral blood [6]. Reportable IG\% enables rapid diagnosis of severe infections and inflammation, reduces the number of microscopic examinations and thus reduces costs, alleviates the workload, and facilitates the follow-up period of the treatment. Early diagnosis of bacteremia and sepsis can reduce morbidity, mortality, and health costs by initiating antimicrobial therapy $[2,7]$.

The goal of the body reactions after an infection is to reduce tissue damage, isolate and eliminate infective organisms, and activate repair mechanisms. The acute phase response is characterized by fever, leukocytosis, and markedly increased levels of some plasma proteins. The acute phase response reflects the presence, activity, and prevalence of the underlying pathology. The monitoring of acute-phase reactants, therefore, provides an objective criterion for disease severity and treatment effectiveness. In addition to biochemical parameters, such as CRP and procalcitonin, some complete blood count (CBC) parameters (leukocytes count, neutrophil count, lymphocyte count, red blood cell distribution width (RDW), platelet count (PLT), platelet hematocrit (PCT), platelet distribution width (PDW) and platelet-large cell ratio (P-LCR)) and indices, such as Platelet-to-Lymphocyte Ratio (PLR), Neutrophil-to-Lymphocyte Ratio (NLR), and Systemic Immune-Inflammatory Index (SII) levels are also given as a hallmark of inflammation [8].

In the present study, we aimed to investigate the role of IG\% and other inflammatory CBC parameters and indices in the inflammation and to assess their correlation. To obtain their prognostic values in detecting inflammation, we also aimed to calculate the sensitivity and specificity values of IG\% and other CBC parameters and indices.

\section{Materials and Methods}

\section{Study design}

We obtained hematological and biochemical data of the 161 outpatients according to their CRP levels, who were admitted to the Koc University Hospitals' outpatient clinic between March 2019 and May 2019 from the database of the hospital laboratory information system in the final study cohort. The exclusion criteria were age younger than 18 years old, a history of recorded coagulopathy or hemolysis in patients' sample data, a diagnosis of chronic disease including diabetes, obesity, chronic renal failure, autoimmune disease, the presence of a hematologic or oncologic disease, pregnancy, having any medication and the presence of a bacterial infection. We made a diagnosis of a bacterial infection depending on a positive bacterial test from any specimen (blood, urine, stool, and pus), the presence of evidence on radiological evaluation, and having clinical findings of a bacterial infection on examination. Patients were administered as having a diagnosis of bacterial infection when a clinical finding was positive (for example, a painful, red infection which is usually warm on the legs for the cellulitis) and the cause might be bacterial (for example, sinusitis, tonsillitis, epiglottitis, mastoiditis, otitis media, lymphadenitis, bacterial urinary tract infection, balanitis, epididymitis, abscess, folliculitis, cellulitis, impetigo or wound infection). Besides, we excluded patients with a WBC count of less than $3.5^{*} 10^{9} / \mathrm{L}$ or more than $10.0^{*} 10^{9} / \mathrm{L}$ regarding potential viral infection or confounding factors. Patients were included in this study if data of all biomarkers (IG\%, CRP, procalcitonin, and hemogram parameters) were available.

To investigate the role of IG\%, procalcitonin, and other hemogram-related inflammatory parameters and indices, patients were classified into three groups according to their CRP level following the criteria described by Chandrashekara et al. [9]. Group I had a CRP-value of $<3 \mathrm{mg} / \mathrm{L}$ (non-inflammatory group)( $\mathrm{n}=58)$, Group II had a CRP level between 3 to $9 \mathrm{mg} / \mathrm{L}$ (low-grade inflammatory group) ( $\mathrm{n}=59)$, and Group III had a CRP level of $>9 \mathrm{mg} / \mathrm{L}$ (clinically significant inflammatory group) ( $n=44)$.

The laboratory data were collected on the first sampling after admission to the outpatient clinic and before medication. Blood samples that were collected into K2EDTA-anticoagulated tubes and processed within two hours were selected for the evaluation. The levels of IG\% and other hemogram parameters were assessed using Sysmex XN-3100 (Sysmex, Kobe, Japan). CRP and procalcitonin were evaluated through the Roche Cobas-6000 analyzer (Roche, Mannheim, Germany).

The between-group difference was evaluated concerning sex, age, CRP, IG\% procalcitonin, leukocyte count, lymphocyte count, neutrophil count, RDW, platelet count, P-LCR, PCT, PDW, platelet-to-lymphocyte ratio, neutrophil-to-lymphocyte ratio and systemic immune-inflammatory index. The SII was defined as follows: SII: platelet count* neutrophil count/lymphocyte count according to the formula described by $\mathrm{Bo} \mathrm{Hu}$ et al. [10].

The present study protocol was reviewed and approved by the Institutional Review Board of Koc University (Approval No. 2019.091.IRB2.028).

The committee exempted in-formed consent regarding the retrospective design of this study.

\section{Statistical analysis}

Statistical analysis was performed using the MEDCALC ${ }^{\oplus}$ v10.2.0 software (MedCalc, Ostende, Belgium). Shapiro-Wilk's test investigated the normality of continuous variables. Descriptive statistics were presented using mean and standard deviation for normally distributed variables and median (interquartile range) for the non-normally distributed variables. 
Non-parametric statistical methods were used for values with skewed distribution. To assess the between-group difference of normally distributed data, the one-way ANOVA test with Tukey's post hoc test for multiple comparisons was performed. Between-group differences of abnormally distributed data were evaluated by performing the Kruskal-Wallis test with Dunn's post hoc test for multiple comparisons. ROC analysis was performed to evaluate the cut-off point for risk factors. Pearson correlation coefficient was used to evaluate the correlation between two normally distributed continuous variables. Spearman correlation coefficient was used to evaluate the correlation between two non-normally distributed continuous variables. Statistical significance was accepted when a two-sided $p$-value was lower than 0.05 .

\section{Results}

In this study 161 outpatients' laboratory data were evaluated. There was no significant between-group difference concerning sex, age, leukocytes count, lymphocyte count, neutrophil count, P-LCR, PCT, PDW and RDW.

In the low-grade inflammatory group (Group II), the levels of CRP $(p<0.0001)$, platelet count $(p<0.05)$, PLR $(p<0.05)$, NLR $(p<0.05)$ and SII $(p<0.01)$ were significantly higher than those in the non-inflammatory group (Group I). In the clinically significant inflammatory group (Group III), CRP ( $p<0.0001)$, IG\% $(p<0.0001)$, procalcitonin $(p<0.01)$, platelet count $(p<0.05)$, PLR $(p<0.01)$, NLR $(p<0.05)$ and SII $(p<0.01)$ values showed a significant between-group difference when compared to
Group I. A significant difference between Group II and Group III was detected for, CRP $(p<0.0001)$ and IG\% $(p<0.05)$, (Table 1$)$.

Regarding correlations of the IG\% with the inflammatory parameters (including CRP, procalcitonin, leukocyte count, platelet count, NLR, PLR and SII), there were significant positive correlations among IG\% and CRP $(p<0.001)$, platelet count $(p<005)$, PLR $(p<0.05)$ and SII $(<0.05)$. Moreover, CRP was the independent predictor ( $\beta=0.427, \mathrm{p}<0.0001$ ) for IG\% (Table 2 ).

Area under the curves (AUC) values from ROC curve analysis for SII, IG\%, PLR, PLT, NLR and procalcitonin were 0.705 (95\% Cl: $0.617-0.783), 0.699$ (95\% Cl: 0.611-0.778), 0.690 (95\% Cl: $0.601-0.769), 0.665$ (95\% Cl: 0.575-0.747), 0.661 (95\% Cl: $0.571-$ 0.744 ) and 0.634 (95\% Cl: 0.543-0.718), respectively (Fig. 1). Immature granulocyte had a sensitivity and specificity value of $75.3 \%$ and $52.5 \%$ respectively (Table 3 ).

\section{Discussion}

In the present study, we found a significant increase in IG\%, procalcitonin, platelet count, PLR, NLR and SII in the inflammatory groups (Group II and III) compared with the non-inflammatory group (Group I). Besides, a notable correlation between IG\% and CRP, SII, PLR and platelet count was noted.

The bone marrow regulates the production of immature granulocytes in response to inflammatory signals. Although the mechanisms of this regulation are obscure, IG\% has been considered as a new predictive $C B C$ parameter to catch the inflammation or any bacterial infection $[8,11]$. Briggs et al. [12] investigated the potential clinical usefulness of positive IG\% (>2\%)

Table 1. Summary characteristics of the total data set according to CRP level

\begin{tabular}{|c|c|c|c|}
\hline Parameters & $\begin{array}{l}\text { Group } 1 \\
\text { (CRP } \leq 3 \mathrm{mg} / \mathrm{L}) \\
\mathrm{n}=58\end{array}$ & $\begin{array}{l}\text { Group } 2 \\
\text { (CRP; } 3-9 \mathrm{mg} / \mathrm{L} \text { ) } \\
\mathrm{n}=59\end{array}$ & $\begin{array}{l}\text { Group } 3 \\
(C R P \geq 9 \mathrm{mg} / \mathrm{L})) \\
\mathrm{n}=44\end{array}$ \\
\hline Age (years) & $61.5(23-88)$ & $62(22-81)$ & $63.5(25-85)$ \\
\hline C-reactive protein (mg/L) & $1.80(0.82-2.70)$ & $7.30(6.10-8.15) a^{* * * *}$ & $13.3(9.83-16.0) b^{* * * *} c^{* * * *}$ \\
\hline Leukocytes count $\left(10^{9} / \mathrm{L}\right)$ & $6.41 \pm 1.77$ & $7.14 \pm 1.64$ & $7.03 \pm 1.81$ \\
\hline Lymphocyte count $\left(10^{9} / \mathrm{L}\right)$ & $1.90(1.40-2.40)$ & $1.70(1.40-2.10)$ & $1.55(1.23-2.10)$ \\
\hline Neutrophil count $\left(10^{9} / \mathrm{L}\right)$ & $3.45(2.85-4.73)$ & $4.90(3.30-6.20)$ & $4.30(3.80-5.68)$ \\
\hline Red blood cell distribution width (\%) & $43.0(41.0-47.9)$ & $43.7(41.0-47.2)$ & $45.6(42.1-50.6)$ \\
\hline Platelet count $\left(10^{9} / \mathrm{L}\right)$ & $224(186-259)$ & $258(213-357) a^{*}$ & $269(214-356) b^{*}$ \\
\hline Platelet-to-lymphocyte ratio (PLR) & $119(94.4-149)$ & $160(119-225) a^{*}$ & $197(116-260) b^{* *}$ \\
\hline Neutrophil-to-lymphocyte ratio (NLR) & $1.92(1.60-2.86)$ & $2.50(2.14-3.79) a^{*}$ & $2.79(1.92-4.20) b^{*}$ \\
\hline Systemic immune inflammatory index (SII) & $470(326-580)$ & $696(497-1113) a^{* *}$ & $778(524-1162) b^{* *}$ \\
\hline
\end{tabular}

a: group 1 vs 2, b: group 1 vs 3, c: group 2 vs $3,{ }^{*} p<0.05,{ }^{* *} p<0.01,{ }^{* * *} p<0.001,{ }^{* * * *} p<0.0001$. The one-way ANOVA test with Tukey's post hoc test or the Kruskal-Wallis test with Dunn's post hoc test for multiple comparisons was performed. Age data were presented as minimum-maximum. Results were expressed as mean \pm SD or median (interquartile range) 
Table 2. Correlations of the immature granulocyte percentage with the inflammatory hemogram parameters and procalcitonin levels

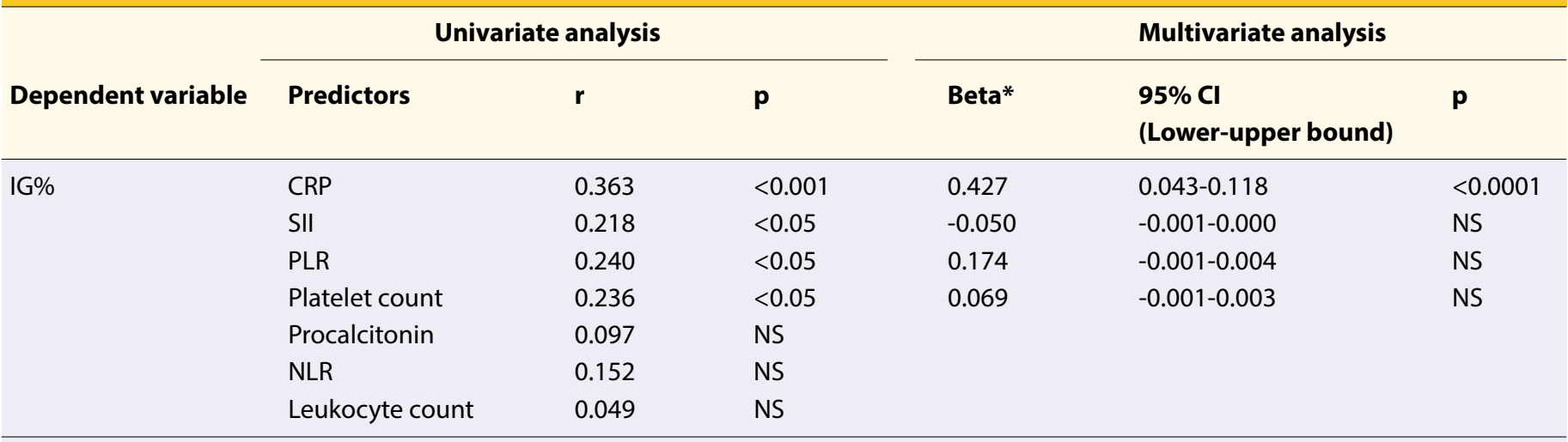

IG\%: Immature granulocyte percentage, CRP: C-reactive protein, SII: Systemic immune inflammatory index, PLR: Platelet-to-lymphocyte ratio NLR: Neutrophil-to-lymphocyte ratio, NS: non-significant. *Standardized beta coefficient

Table 3. The areas under curve, sensitivity and specificity values of IG\%, procalcitonin, platelet count, PLR, NLR and SII

\begin{tabular}{llllll}
\hline & AUC 95\% CI & p & Cut off & Sensitivity (95\% CI) & Specificity (95\% CI) \\
\hline Immature granulocyte percentage (\%) & $0.699(0.611-0.778)$ & 0.0001 & $>0.2$ & $75.3(64.7-84.0)$ & $52.5(36.1-68.5)$ \\
Procalcitonin $(\mathrm{ng} / \mathrm{mL})$ & $0.634(0.543-0.718)$ & 0.0093 & $>0.05$ & $56.5(45.3-67.2)$ & $62.5(45.8-77.3)$ \\
Platelet count (10\%) & $0.665(0.575-0.747)$ & 0.0009 & $>268$ & $47.1(36.1-58.2)$ & $82.5(67.2-92.6)$ \\
Platelet-to-lymphocyte ratio (PLR) & $0.690(0.601-0.769)$ & 0.0001 & $>170$ & $49.4(38.4-60.5)$ & $87.5(73.2-95.8)$ \\
Neutrophil-to-lymphocyte ratio (NLR) & $0.661(0.571-0.744)$ & 0.0012 & $>2.11$ & $74.1(63.5-83.0)$ & $60.0(43.3-75.1)$ \\
Systemic immune inflammatory index (SII) & $0.705(0.617-0.783)$ & 0.0001 & $>512$ & $74.1(63.5-83.0)$ & $67.5(50.9-81.4)$
\end{tabular}

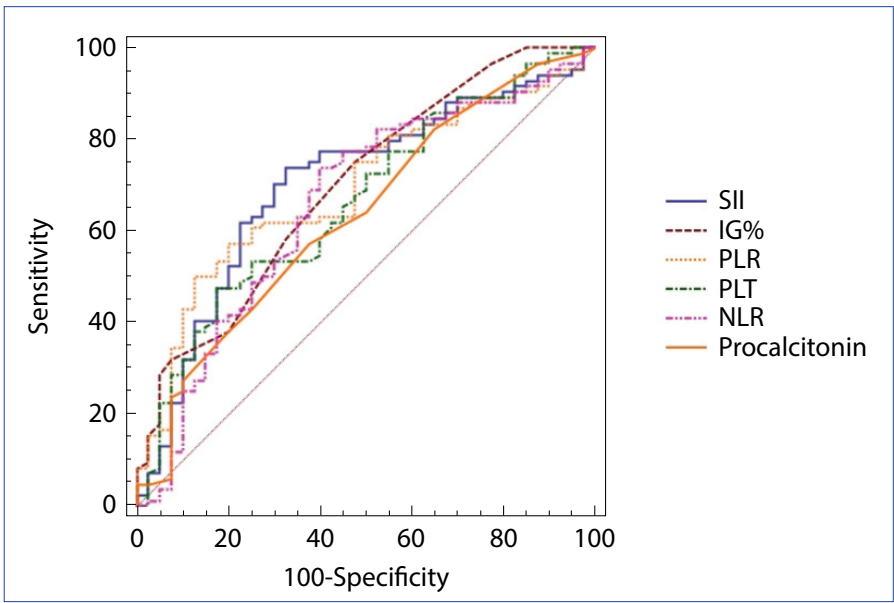

Figure 1. Area under the curves (AUC) values from ROC curve analysis for SII, IG\%, PLR, PLT, NLR and procalcitonin were 0.705 (95\% Cl: 0.6170.783), 0.699 (95\% Cl: 0.611-0.778), 0.690 (95\% Cl: 0.601-0.769), 0.665 (95\% Cl: $0.575-0.747), 0.661$ (95\% Cl: $0.571-0.744)$ and 0.634 (95\% Cl: $0.543-0.718)$, respectively.

ROC: Receiver operating characteristics; IG\%: Immature granulocytes percentage; NLR: Neutrophil-to-lymphocyte ratio; PLR: Platelet-to-lymphocyte ratio; SII: Systemic immune inflammatory index (SII: Platelet count* neutrophil count/ lymphocyte count) and PLT: platelet count.

in blood counts with a normal neutrophil count by evaluating random samples. The level of CRP $(p=0.0090)$ and erythrocyte sedimentation rate $(p=0.001)$ were significantly higher in the positive IG group than those in the control group (IG\%<2\%). They demonstrated that $28.6 \%$ of the patients with a positive IG count were from a proven or unsuspected developing acute infectious period. The remaining IG\%-positive samples were from patients with various acute pathologic situations, such as post-operation, acute and chronic blood loss, or renal failure. All of these conditions are likely to be associated with an acute-phase response in which activated chemotactic factors and cytokines stimulate the bone marrow to release IG\% into the circulation. In the present study, we detected significantly higher values of IG\% in Group III than in the Group I and II ( $p<0.0001$ and $p<0.05$, respectively). However, the absence of a significant difference between Group 1 and 2 made us think that as the severity of inflammation increases, IG\% can become more valuable in the diagnosis.

CRP and procalcitonin are biochemical markers of inflammation and infection. CRP is synthesized in hepatocytes following stimulation by pro-inflammatory cytokines and secreted into the blood in response to inflammation and infection [13]. In this study, for CRP, the three groups showed a significant difference as pre-determined. The procalcitonin values were significantly higher in Group III $(p<0.01)$ compared to Group I. Platelets have a vital role in various inflammatory diseases by interacting with most immune cells. Several factors are synthesized at the surface of platelets or are captured in a-granules lead platelets to react to systemic inflammation and 
support immune responses. By the overproduction of pro-inflammatory cytokines, chronic inflammation leads to reactive thrombocytosis [14]. We obtained significantly higher platelet count in Group II $(p<0.05)$ and III $(p<0.05)$ when compared to the Group I in line with Ustundag et al. [15]. However, no significant difference was found between inflammatory groups, which may be associated with cytokine release, reactive thrombocytosis, or other confounding factors.

Inflammatory ratios, including lymphocytes (PLR, NLR and SII), are less affected by interfering situations than other leukocyte parameters or routinely used inflammatory markers [16]. They have been investigated as novel inflammatory markers that can be used to investigate systemic inflammation in various diseases [17]. In this study, PLR and NLR were found to be significantly higher in the Group II ( $\mathrm{P}<0.05$ for both) and III $(\mathrm{P}<0.01$ and $P<0.05$ for PLR and NLR, respectively) when compared to the Group I, in line with Mazza et al. [18]. However, no significant difference was detected between Group I and Group II, which shows that the predictive ability of PLR and NLR may be lower at the beginning of the inflammation. Another novel inflammatory index, SIl, which is thought to have strong prognostic value, shows the local immune response and systemic inflammation [10, 19]. The SII index has been recently reported to be associated with poor outcome in patients with hepatocellular and colorectal carcinoma [20]. In the present study, SII was significantly higher in Group II $(p<0.01)$ and III $(p<0.01)$ when compared to Group I.

In the univariate analysis, we found a significant correlation between IG\% and CRP $(r=0.363, p<0.001)$, platelet count $(r=0.236, p<0.05)$, PLR $(r=0.240, p<0.05)$ and SII $(r=0.218$, $\mathrm{p}<0.05)$. Furthermore, CRP was the independent predictor ( $\beta=0.427, p<0.0001$ ) for IG\% (Table 2 ). There was no significant correlation between IG\% and leukocyte count, procalcitonin and NLR, which may be due to the low number of the study population. Hence, further studies are needed to investigate the correlation analysis between IG\% and other biomarkers with larger populations.

We performed ROC curves of IG\%, procalcitonin, platelet count, PLR, NLR, and SII (Fig. 1). The predictive ability of SII was better than IG\%, procalcitonin, platelet count, PLR and NLR with an area under the curve value of 0.705 (95\% Cl: 0.6170.783). The sensitivity and specificity values of the IG\% using a cut-off value of $>0.2$ were $75.3 \%$ and $52.5 \%$, respectively, in predicting inflammation (Table 3 ). In contrast to our results, Briggs et al. [12] reported a high value of specificity (83\%) with a low value of sensitivity (36.2\%) for IG count in detecting inflammation. In another study, Ansari-Lari et al. [2] reported a better correlation with IG\% in patients with infection and positive blood cultures than leukocyte count, even the sensitivity was low (\%40 sensitivity, 90\% specificity). Many studies have evaluated a broad range (ranging from 30\% to 95\%) for the sensitivity and specificity values of IG\% in various diseases [21-23]. This considerable sensitivity and specificity discrepancies may be due to the severity of inflammation, the age and number of the study population or the presence of concomitant disease in selected patients.

In the present study, we aimed to see the role of IG\% and inflammatory hemogram indices to investigate the severity of inflammation, evaluate their correlation, and assess their predictive ability in classifying inflammation. Our study showed that to detect the severity of inflammation, it would be more reliable to evaluate the combination of these parameters (IG\%, PLR, NLR, SII) instead of looking at a single one.

There were three limitations to our study. First, a selection bias might occur due to the studies' retrospective design. The study population may have been classified according to a particular disease. Second, we did not evaluate IG\% in other equipment. Hence, study results should be validated on another analyzer before clinical use. Third, we tried to exclude bacterial infection from all study groups. However, according to CRP and procalcitonin levels, it may not fully be achieved in Group 3. The presence of infection in addition to inflammation could be a confounding factor in interpreting the results.

\section{Conclusion}

This study showed that increased levels of IG\%, procalcitonin, platelet count, PLR, NLR and SII might play a role in the classification of inflammation. CRP is quick, readily available and cheap to perform. However, it lacks specificity as an inflammatory marker. Combining CRP with IG\% and other inflammatory hemogram indices may help detect the samples in patients in whom bone marrow stimulation appears at an early stage as part of the acute-phase response to an infection or inflammation. Further prospective studies with many patients are needed to validate the clinical usefulness of IG\% and other hematological inflammatory markers.

Conflict of Interest: The researchers identified no potential conflict of interest with any entity regarding the content discussed.

Ethics Committee Approval: The present study protocol was reviewed and approved by the Institutional Review Board of Koc University (Approval No. 2019.091.IRB2.028).

Financial Disclosure: None declared.

Peer-review: Externally peer-reviewed.

Authorship Contributions: Concept - S.I., K.E.P.; Design - S.I., K.E.P.; Supervision - S.I., K.E.P.; Funding - None; Materials - S.I., K.E.P.; Data collection \&/or processing - H.K.C., S.I.; Analysis and/ or interpretation - S.I., H.K.C.; Literature search - H.K.C.; Writing S.I., K.E.P.; Critical review - S.I., K.E.P.

\section{References}

1. Nigro KG, O'Riordan M, Molloy EJ, Walsh MC, Sandhaus LM. Performance of an automated immature granulocyte count as a predictor of neonatal sepsis. Am J Clin Pathol 2005;123(4):618-24. [CrossRef] 
2. Ansari-Lari MA, Kickler TS, Borowitz MJ. Immature granulocyte measurement using the Sysmex XE-2100. Relationship to infection and sepsis. Am J Clin Pathol 2003;120(5):795-9. [CrossRef]

3. Ha SO, Park SH, Park SH, Park JS, Huh JW, Lim CM, et al. Fraction of immature granulocytes reflects severity but not mortality in sepsis. Scand J Clin Lab Invest 2015;75(1):36-43.

4. Cornbleet PJ. Clinical utility of the band count. Clin Lab Med 2002;22(1):101-36. [CrossRef]

5. Cimenti C, Erwa W, Herkner KR, Kasper DC, Müller W, Resch $B$. The predictive value of immature granulocyte count and immature myeloid information in the diagnosis of neonatal sepsis. Clin Chem Lab Med 2012;50(8):1429-32. [CrossRef]

6. Kong T, Park YS, Lee HS, Kim S, Lee JW, Yu G, et al. Value of the Delta Neutrophil Index for Predicting 28-Day Mortality in Patients With Acute Pulmonary Embolism in the Emergency Department. Shock 2018;49(6):649-57. [CrossRef]

7. Briggs C, Harrison P, Grant D, Staves J, MacHin SJ. New quantitative parameters on a recently introduced automated blood cell counter--the XE 2100. Clin Lab Haematol 2000;22(6):34550. [CrossRef]

8. Henriot I, Launay E, Boubaya M, Cremet L, Illiaquer M, Caillon $\mathrm{H}$, et al. New parameters on the hematology analyzer $\mathrm{XN}-10$ (SysmexTM) allow to distinguish childhood bacterial and viral infections. Int J Lab Hematol 2017;39(1):14-20. [CrossRef]

9. Chandrashekara S. C - reactive protein: An inflammatory marker with specific role in physiology, pathology, and diagnosis. Internet J Rheumatol Clin Immunol 2014;2(S1):1-23.

10. Hu B, Yang XR, Xu Y, Sun YF, Sun C, Guo W, et al. Systemic immune-inflammation index predicts prognosis of patients after curative resection for hepatocellular carcinoma. Clin Cancer Res 2014;20(23):6212-22. [CrossRef]

11. Ueda $Y$, Kondo M, Kelsoe $G$. Inflammation and the reciprocal production of granulocytes and lymphocytes in bone marrow. J Exp Med 2005;201(11):1771-80. [CrossRef]

12. Briggs C, Kunka S, Fujimoto H, Hamaguchi $Y$, Davis BH, Machin SJ. Evaluation of immature granulocyte counts by the XE-IG master: upgraded software for the XE-2100 automated hematology analyzer. Lab Hematol 2003;9(3):117-24.

13. Späth C, Srinivasa S, Walsh M, Singh P, Rodgers M, Koea J. Role of post-operative serum C-reactive protein levels as a predic- tor of complications in upper gastrointestinal surgery. ANZ J Surg 2019;89(1-2):74-8. [CrossRef]

14. Müller-Newen G, Stope MB, Kraus T, Ziegler P. Development of platelets during steady state and inflammation. J Leukoc Biol 2017;101(5):1109-17. [CrossRef]

15. Ustundag Y. Relationship between C-reactive protein, systemic immune-inflammation index, and routine hemogramrelated inflammatory markers in low-grade inflammation. Int J Med Biochem 2018;1(1):24-8. [CrossRef]

16. Gibson PH, Croal BL, Cuthbertson BH, Small GR, Ifezulike Al, Gibson $\mathrm{G}$, et al. Preoperative neutrophil-lymphocyte ratio and outcome from coronary artery bypass grafting. Am Heart J 2007;154(5):995-1002. [CrossRef]

17. Can E, Hamilcikan Ş, Can C. The Value of Neutrophil to Lymphocyte Ratio and Platelet to Lymphocyte Ratio for Detecting Early-onset Neonatal Sepsis. J Pediatr Hematol Oncol 2018;40(4):e229-32. [CrossRef]

18. Mazza MG, Lucchi S, Rossetti A, Clerici M. Neutrophil-lymphocyte ratio, monocyte-lymphocyte ratio and platelet-lymphocyte ratio in non-affective psychosis: A meta-analysis and systematic review. World J Biol Psychiatry 2020;21(5):326-38.

19. Hong X, Cui B, Wang M, Yang Z, Wang L, Xu Q. Systemic Immune-inflammation Index, Based on Platelet Counts and Neutrophil-Lymphocyte Ratio, Is Useful for Predicting Prognosis in Small Cell Lung Cancer. Tohoku J Exp Med 2015;236(4):297-304.

20. Lolli C, Caffo O, Scarpi E, Aieta M, Conteduca V, Maines F, et al. Systemic Immune-Inflammation Index Predicts the Clinical Outcome in Patients with mCRPC Treated with Abiraterone. Front Pharmacol 2016;7:376. [CrossRef]

21. Nierhaus A, Klatte S, Linssen J, Eismann NM, Wichmann D, Hedke J, et al. Revisiting the white blood cell count: immature granulocytes count as a diagnostic marker to discriminate between SIRS and sepsis--a prospective, observational study. BMC Immunol 2013;14:8. [CrossRef]

22. Lee JW, Kim SH, Park SJ, Lee KH, Park JH, Kronbichler A, et al. The value of delta neutrophil index in young infants with febrile urinary tract infection. Sci Rep. 2017;7:1-9. [CrossRef]

23. Park HJ, Ha YJ, Pyo JY, Park YB, Lee SK, Lee SW. Delta neutrophil index as an early marker for differential diagnosis of adult-onset Still's disease and sepsis. Yonsei Med J 2014;55(3):753-9. 